ÉGYPTE monde arabe

\section{Égypte/Monde arabe}

$34 \mid 1998$

Droits d'Égypte : histoire et sociologie

\title{
Négociation et écriture
}

À propos du droit coutumier en Haute-Égypte

\section{Hans Christian Korsholm Nielsen}

Traducteur : Kjerstin Vedel

\section{Q OpenEdition}

\section{Journals}

Édition électronique

URL : https://journals.openedition.org/ema/1517

DOI : 10.4000/ema. 1517

ISSN : 2090-7273

\section{Éditeur}

CEDEJ - Centre d'études et de documentation économiques juridiques et sociales

\section{Édition imprimée}

Date de publication : 31 décembre 1998

Pagination : 155-165

ISSN : 1110-5097

\section{Référence électronique}

Hans Christian Korsholm Nielsen, « Négociation et écriture », Égypte/Monde arabe [En ligne], 34 | 1998, mis en ligne le 08 juillet 2008, consulté le 07 juillet 2022. URL : http://journals.openedition.org/ema/ 1517 ; DOI : https://doi.org/10.4000/ema.1517

Ce document a été généré automatiquement le 7 juillet 2022

Tous droits réservés 


\title{
Négociation et écriture
}

\author{
À propos du droit coutumier en Haute-Égypte
}

Hans Christian Korsholm Nielsen

Traduction : Kjerstin Vedel

\section{NOTE DE L'ÉDITEUR}

Ce texte a été traduit du danois par Kjerstin Vedel, maître-assistant à l'université de Aarhus, Danemark

1 Les descriptions anthropologiques du droit coutumier ('urf)au Moyen-Orient sont souvent marquées par les conditions de la recherche, qui s'est focalisée sur la situation particulière des bédouins du désert, ou a tendu à faire croire que le droit coutumier n'est que le vestige exotique d'un passé lointain. Moins nombreuses sont les études qui s'occupent des conditions de vie des sédentaires et encore moins celles qui traitent du droit coutumier comme d'une partie intégrante de la société moderne ${ }^{1}$. À partir d'environ cinquante documents rédigés à la suite du prononcé de jugements par des « assemblées de réconciliation » dans la région d'Edfou en Haute-Égypte, je tâcherai de démontrer que le droit coutumier n'est pas un vestige archaïque, mais bien un phénomène vivant qui exige de ses acteurs la possession d'un savoir spécifique permettant la conduite d'une négociation et l'obtention d'une décision, de même que la connaissance des procédures de rédaction des documents et de contrôle du respect des décisions dans une société en profonde transformation.

Lorsqu'un conflit surgit entre les habitants d'un village ou d'une ville en Haute-Égypte et qu'il se complique au point que les parties jugent nécessaire - ou se voient obligées d'avoir recours à des instances extérieures pour arriver à la solution de leurs problèmes, il y a deux institutions à la fois différentes et complémentaires auxquelles elles peuvent s'adresser. D'un côté, un conflit peut être déféré au tribunal officiel d'Edfou. Le cas est fréquent, comme en témoigne l'activité frénétique et permanente autour du palais de justice. D'un autre côté, la solution des conflits peut être recherchée 
par l'entremise d'une "assemblée de réconciliation» propre au système juridique coutumier.

3 En Haute-Égypte ces systèmes sont connus sous des noms différents, à savoir : majlis alsulh, majlis 'urfí majlis al-'arab². Chacune de ces dénominations fait référence à certaines qualités du système juridique coutumier : il s'agit d'une assemblée (majlis)qui, par la négociation, doit parvenir à un accord (sulh) en fondant ses décisions sur la coutume ('urf)et non pas, autrement dit, sur la loi islamique (sharî'a) ou la législation positive. Il est toutefois courant d'insister sur l'équivalence entre le 'urf et la sharî' $a^{3}$ ou du moins sur le fait que l'un prolonge l'autre, le 'urf concrétisant certaines dispositions édictées dans la loi religieuse. Ainsi en va-t-il, par exemple, de l'importance et de la nature de l'amende compensatoire prescrite par la sharî'a. Les mandats trouvent de manière non explicite leur fondement juridique dans la coutume. Le savoir traditionnel portant sur les prescriptions et les pratiques de négociation est transmis d'un individu à l'autre par la participation aux assemblées, en qualité de membre ou d'auditeur. Globalement parlant, il n'existe que peu d'exemples de prescriptions standardisées ou de situations où la solution correcte est connue d'avance.

4 À la question de savoir ce qu'est le 'urf, on se voit répondre par la description de tout le processus, y compris la négociation et la réunion publique de l'assemblée. Il est rare que les gens vous expliquent les règles de compensation, que ce soit pour blessure, atteinte à des animaux domestiques ou à d'autres propriétés. Le 'urf peut être idéalement décrit comme un discours pratique qui s'exprime à travers les déclarations et les décisions des assemblées. La description du contenu ou des modes constitutifs de ces décisions demanderait de lire de nombreux cas qui tous paraissent plus spécifiques les uns que les autres. Comme le dit Pierre Bourdieu, «le droit coutumier semble toujours aller du cas particulier au cas particulier, de l'acte spécifique à la sanction spécifique, sans jamais formuler les principes fondamentaux que le droit « rationnel » mentionne explicitement » (Bourdieu, 1977, p. 17).

Le savoir ainsi accumulé est en même temps étroitement lié aux hommes qui participent activement aux assemblées en tant que conciliateurs. Il est " homéostatique ", pour reprendre la terminologie de Goody et Watt (1963), et non pas couché dans un texte juridique. La crise qui est provoquée par un conflit rend visible une relation spécifique dans la société locale et la plus importante fonction de l'assemblée est idéalement de formuler une solution fondée sur un savoir qui est commun mais pas articulé. Dans la terminologie de Bourdieu, les membres de l'assemblée doivent « éveiller ».

6 L'appellation majlis al-'arab fait référence aux assemblées tribales qui se tenaient à une époque légendaire dans la péninsule Arabique et au fait que la majorité de la population de Haute-Égypte estime descendre des tribus arabes qui ont immigré en Égypte au VIle siècle. Lorsqu'une solution a été trouvée par une assemblée de ce type pour régler un conflit, il est de coutume de rédiger un document présentant les membres de l'assemblée, les parties en conflit, les faits centraux ainsi que la conclusion de l'assemblée. Ces documents portent le plus souvent le titre de mahdar sulh (procèsverbal de conciliation) ou de mahdar tahkîm (procès-verbal de négociation).

7 Cet article s'appuie sur ma participation à un certain nombre de ces assemblées de réconciliation ainsi que sur une collection d'environ cinquante documents rédigés à l'occasion du prononcé des jugements. Ces documents ont été rassemblés au cours des recherches de terrain que j'ai menées à Edfou entre 1994 et 1997. 


\section{Une région en Haute-Égypte}

8 La région d'Edfou, située au nord d'Assouan, regroupe environ 350000 habitants dont 70000 pour la seule ville d'Edfou. C'est une région agricole, spécialisée principalement dans la production de canne à sucre. En dépit de certaines initiatives ayant visé, ces dernières années, à assurer une plus grande diversité dans le secteur agricole, la production sucrière reste dominante. L'agriculture se fait, à l'image de toute l'agriculture égyptienne, par arrosage. Depuis la construction du barrage d'Assouan, l'arrosage se fait grâce au pompage de l'eau et à un système de canaux, ce qui permet aujourd'hui plusieurs récoltes par an. Auparavant, l'agriculture suivait le rythme des crues du Nil et la récolte se faisait une seule fois l'an. Depuis le début des années soixante, la production agricole est donc en forte expansion.

Dans la région, une usine de sucre emploie en haute saison un grand nombre de personnes. On trouve également d'autres productions industrielles importantes fondées sur le raffinement de matières premières (le phosphate, par exemple) qui sont extraites dans les régions désertiques à l'est et à l'ouest de la vallée du Nil. De par sa position de ville principale de la région, Edfou est le siège d'un grand nombre d'institutions publiques: hôpital, institutions éducatives, siège des postes et télécommunications. Ces institutions emploient également un grand nombre de personnes. En même temps, la ville, qui se situe sur le parcours des bateaux reliant Louxor à Assouan, bénéficie d'importantes rentrées d'argent grâce au tourisme.

10 La société est divisée en multiples tribus dont les plus connues et les plus nombreuses sont la Ja'âfra et la 'Abâbda. Les membres de la première se comptent parmi les descendants de Muhammad et les seconds disent descendre de Zubayr Ibn al-'Awâm, une branche moins prestigieuse de la tribu de Quraysh. Plus de $90 \%$ de la population est musulmane malikite, le reste appartenant à l'église copte orthodoxe.

\section{Conciliation et négociation}

11 Quand c'est par l'intermédiaire des assemblées de négociation traditionnelles que l'on cherche à résoudre le conflit, le processus est initié par quelques hommes ou par un groupe d'hommes qui s'adressent aux parties en conflit pour proposer une solution. L'initiative est toujours prise par des personnes extérieures au conflit. S'il s'agit d'un conflit entre les membres d'une même famille, les conciliateurs sont des personnes extérieures à celle-ci ; s'agit-il d'un conflit tribal interne, les personnes qui prennent l'initiative viennent toujours d'une autre tribu; s'agit-il enfin d'un conflit impliquant deux tribus, les conciliateurs sont toujours issu d'une tierce tribu.

12 Par leurs premières initiatives, les conciliateurs cherchent à obtenir que les parties acceptent qu'une solution soit recherchée. En partant du principe qu'il est indigne pour une famille et contraire à l'idéal du bon voisinage d'être publiquement en conflit, ils cherchent à amener les parties à la négociation. Cela signifie que les deux parties ne participent pas directement au déroulement des négociations. Les conciliateurs pratiquent une sorte de diplomatie de la navette entre les deux parties, les informant des arguments de leur opposant respectif, des concessions que l'assemblée juge nécessaire d'accepter et du point où en est le processus. À un moment donné, lorsque 
les conciliateurs estiment que les négociations sont suffisamment avancées pour qu'une solution soit possible, une assemblée réelle, qui devra prononcer un jugement, est constituée. En principe, les membres de l'assemblée doivent être élus et acceptés par les deux parties. Souvent, cela fait l'objet de discussions intenses. Chaque partie propose un certain nombre de candidats qui doivent ensuite êtres acceptés par l'opposant qui, à son tour, a le droit de proposer un certain nombre de candidats. En cas de refus des noms proposés, la partie a le droit de désigner d'autres candidats. Normalement, cette négociation ne se déroule pas d'une façon aussi systématique, mais il est important que les membres de l'assemblée soient acceptés par les deux parties. L'assemblée doit en tout cas être impartiale et ses membres ne doivent pas pouvoir profiter de ce qui en résultera. Le prestige et le statut qui peuvent être acquis en étant élu membre d'une assemblée n'entrent pas ici en ligne de compte.

Un acte de procuration (mahdar tafwîd)est alors rédigé et signé par les parties. Par là, elles attestent qu'elles sont prêtes à se soumettre à toute décision prise par l'assemblée. Les parties cèdent à l'assemblée une part des droits liés à leur "personne", dans la mesure où elles renoncent à leur droit d'agir individuellement et de faire intervenir d'autres instances dans le conflit. Ce document indique également le montant de l'amende que l'assemblée peut infliger à la partie qui ne respecte pas sa décision. Il s'agit souvent d'un montant très élevé.

Dans de nombreux cas, l'acte de procuration est intégré dans le document final et n'est signé qu'au moment de la réunion officielle et publique qui se tient à l'occasion de la mise au point de la procédure. Une part de la littérature anthropologique traitant de conciliation s'accorde à dire qu'il y a une distinction nette entre médiation et prononcé de jugement ${ }^{4}$. Pareille distinction est atténuée dans les cas observés en Haute-Égypte. En effet, la première partie du processus - avant la signature de l'acte de procuration relève bien de la négociation (ce à quoi le terme sulh fait indirectement référence). À partir de ce moment, toutefois, c'est l'assemblée qui mène la négociation jusqu'à ce qu'elle parvienne à une décision qui ressemble, sous plus d'un aspect, au prononcé d'un jugement, les parties acceptant par leur signature de n'avoir aucun droit d'opposition ou d'influence sur la décision.

\section{Les documents}

Le résumé des conciliations (les documents de sulh)est normalement manuscrit et édité en trois exemplaires (un exemplaire pour chaque partie et le troisième pour l'assemblée) valant copie conforme. Le texte est le plus souvent rédigé en vue de paraître court, mais contient néanmoins énormément de références aux détails spécifiques de la topographie locale et de la production agricole. Il est nécessaire d'avoir une connaissance détaillée de la région pour pouvoir reconstruire le processus sur la base descriptive des documents. Il s'agit en effet de courtes descriptions d'un processus fondamentalement oral. Normalement, les documents ne sont pas traités avec beaucoup de respect : leur contenu est considéré comme important en tant que preuve de la solution trouvée, mais il n'est pas question de le traiter comme quelque chose d'extraordinaire du fait de sa nature écrite. Ils sont très souvent gardés dans un placard ou un coffre avec d'autres écrits, comme par exemple de vieux cahiers scolaires ou des factures. En principe, les documents sont accessibles au public et il ne paraît pas problématique de les montrer ou d'en donner une copie à un ethnologue de passage. 
L'acte auquel ils font référence a généralement été déjà prononcé lors d'une réunion publique à laquelle assistaient de nombreux hommes de la région.

Le fait de consigner l'acte par écrit est souvent considéré comme le signe d'une évolution récente ${ }^{5}$ due à la mobilité toujours plus grande de la société égyptienne d'aujourd'hui. D'autres phénomènes comme, par exemple, la capacité de lire ou l'influence du système judiciaire officiel et de la bureaucratie égyptienne en général peuvent jouer un rôle : à travers le contact avec les institutions publiques, la population égyptienne a appris à connaître l'importance d'être en possession des bons documents, tampons et signatures.

17 Au sein de la population âgée, on entend souvent dire que la raison pour laquelle on a commencé à rédiger le prononcé des jugements est due à la décadence des mœurs : proximité, respect et honneur, qui sont des phénomènes centraux dans la relation entre les générations en Haute-Égypte, semblent aujourd'hui avoir reflué. Bien que les documents soient secondaires par rapport à la négociation orale et, en tant que tels, faiblement vénérés, ils tiennent malgré tout un rôle important. Brinkley Messick dit qu'ils reflètent «le point de croisement du droit et de ses usages sociaux » (Messick, 1990, p. 61). Ils expriment plutôt les conflits de la tradition ('urf) et de son application sociale. Ils représentent dès lors une documentation unique sur le développement de la société en Haute-Égypte ces vingt dernières années et leur contenu traduit bien les divers conflits qui la traversent et que l'institution de négociation tente de résoudre.

\section{La rédaction des documents}

Une partie à la conciliation (taraf, pl. atrâf)peut être représentée par une personne ou par un petit groupe de proches (frères ou cousins). Dans certains cas, il peut s'agir de négociations entre des quartiers entiers d'un village. Les groupes en conflit sont alors qualifiés de tribus (qabîla, pl. qabấil), même s'ils ne correspondent pas à la définition qu'en donne la littérature anthropologique. Un petit nombre d'hommes est alors choisi pour représenter le groupe. Ce sont les signataires du document et ils sont, par conséquent, responsables devant l'assemblée et devant le groupe.

Les femmes et les mineurs peuvent se faire représenter par un tuteur, mais il est courant que, dans les affaires de succession, les femmes participent indépendamment aux négociations ou y représentent des mineurs. Ce n'est guère surprenant, dès lors que les affaires de succession ne concernent que la famille proche et n'exposent pas la femme à un groupe d'étrangers ${ }^{6}$.

Les premières lignes d'un document sulh indiquent toujours la date et le lieu de la réunion de l'assemblée de négociation. Elles sont suivies d'un paragraphe où les membres de l'assemblée sont présentés avec leurs titres. Leur nombre et leur qualité indiquent la gravité du conflit. Les conflits de sang voient assez souvent la participation du chef de la police et du gouverneur d'Assouan. En principe, toutefois, les membres de l'assemblée peuvent provenir de n'importe quel groupe social. L'assemblée est généralement composée d'un certain nombre d'hommes âgés qui sont renommés pour leur faculté de négociation et leur connaissance de la tradition et des règles du cérémonial entourant le prononcé du jugement. La participation à l'assemblée repose donc, de fait, sur la connaissance de la tradition et sur le statut de la personne. Les membres de l'assemblée peuvent ainsi exercer les fonctions d'avocat auprès du tribunal 
d'Edfou. Toutefois, ce n'est pas en raison de leur savoir professionnel qu'ils y participent, mais uniquement en raison de leur statut dans la société locale.

Dans le deuxième paragraphe, les parties en conflit sont mentionnées (généralement deux, mais rien n'exclut qu'elles soient plus nombreuses). Il peut s'agir d'un individu, d'un groupe (généralement des parents), voire même d'une section entière d'un village.

Le troisième paragraphe du document présente les " faits ", qui sont bien entendu ceux que l'assemblée établit et sanctionne. Ils sont établis au cours des négociations qui ont lieu avant le prononcé de la décision, au moyen d'interviews avec les parties impliquées et de rapports d'experts. Si le conflit porte sur la terre ou sur la propriété, on recourt à un arpenteur pour chercher l'information ou établir la superficie de la terre ou des bâtiments qui sont l'objet du litige. La négociation qui entoure l'établissement des faits est une démarche centrale dans le déroulement du processus. Elle est essentielle aussi bien pour l'assemblée que pour les deux parties. Ce récit est celui sur lequel les parties s'accordent et, bien qu'il ne s'agisse pas de la vérité de l'affaire, il s'agit de la version acceptable pour tous ou, à tout le moins, celle sur laquelle un consensus provisoire a pu être atteint.

Mais il ne suffit pas que l'assemblée parvienne à un accord entre les deux parties sur «ce qui a eu lieu ». Le récit doit également, par la publicité qui lui est donnée, être sanctionné par le public. Un prononcé de jugement par trop en contradiction avec l'idée de ce qui est bon et raisonnable n'est normalement pas accepté et, par voie de conséquence, disqualifie les membres de l'assemblée. L'éventualité d'un rejet de la décision n'est normalement pas mentionnée dans le document. Je n'ai pu observer qu'un seul jugement dans lequel l'assemblée proposait, au dernier paragraphe, que les parties fassent appel de la décision devant une nouvelle assemblée en cas de désaccord.

Le nouveau récit tient sa force du fait qu'il résulte d'un accord entre les parties. Les faits ont été réorganisés et ceci permet de sortir de la situation sans compromission ni défaite humiliante. Dans le processus final, il arrive souvent qu'un membre de l'assemblée s'adresse séparément aux parties pour les convaincre de conclure l'accord. Il semble moins compliqué d'accepter des concessions lorsque cela se fait séparément et à l'abri des regards du public.

Suit le paragraphe qui indique la décision de l'assemblée. Ainsi, par exemple, un terrain doit être divisé, une fenêtre doit être déplacée parce qu'elle porte atteinte à l'intimité d'un voisin, un canal d'arrosage doit être nettoyé, une pompe doit être remplacée ou une amende échue doit être payée, etc. Le paragraphe est suivi de la déclaration stipulant que la décision est valable au regard de la tradition et des autorités officielles, ce qui veut dire qu'elle constitue la décision définitive. En principe, aucune des parties n'a le droit de l'attaquer par la suite devant un tribunal officiel. À moins que cela n'ait été dit préalablement, le paragraphe invite également celui qui a introduit un recours en justice à le retirer. Les sanctions qui accompagnent toute rupture de la décision sont indiquées à nouveau. Généralement, un montant à payer a été fixé et la somme est le plus souvent très élevée. Dans certains cas, les deux parties sont obligées de signer un chèque "en blanc » et l'assemblée fixe le montant de l'amende à verser en cas de rupture. L'assemblée décide également de l'emploi qui sera éventuellement fait de cette somme. Dans certains documents, il est précisé qu'en cas de rupture de la décision, la partie lésée a le droit de recourir devant le tribunal d'Edfou. Dans ce cas, le système juridique officiel, qui est considéré dans certains domaines comme opposé au système arbitral, est explicitement reconnu comme instance dotée d'un pouvoir de sanction. 
Par ailleurs, il est connu qu'en cas de rupture de la décision et de recours à un tribunal officiel, le système judiciaire n'accepte qu'à contrecœur de reprendre le dossier et le plus souvent tranche dans le même sens que l'assemblée.

Les paragraphes qui concluent les documents contiennent souvent des louanges à Dieu et la mention de ce qu'il est le Témoin ultime. Suivent enfin les signatures des membres de l'assemblée et des parties, parfois également des empreintes digitales. Quelques documents sont également tamponnés, souvent par des autorités municipales.

\section{Documentation et négociation}

27 Jusqu'à il y a vingt ou trente ans, les décisions étaient officialisées par leur prononcé en présence d'un groupe d'hommes physiquement, familialement et professionnellement liés à la région et aux parties impliquées. Ainsi, la société locale garantissait, avec les membres de l'assemblée, la soumission des parties à la décision. Aujourd'hui encore, c'est la coutume ('urf), entendue dans un sens large, qui demeure au fondement des décisions et donc de leur acceptation. Mais avec les changements accélérés que connaît la société égyptienne, qui se traduisent par une mobilité croissante - migration rurale, migration vers les États riches du Golfe - une transformation des rapports intergénérationnels et une expansion de l'alphabétisation ${ }^{7}$, le respect des décisions ne peut plus dépendre de la seule société locale.

Le recours à l'écrit semble donc suivre une évolution logique. Il constitue en même temps un bon exemple de l'adaptation d'une tradition locale à des conditions modifiées. La rédaction des décisions semble avoir généré une nouvelle situation qui permet la monopolisation du savoir de la tradition et ainsi la concentration du pouvoir lié à l'exercice du droit coutumier entre les mains d'un groupe de professionnels.

Il peut par ailleurs sembler aller de soi qu'une fois les décisions mises par écrit, c'est toute l'institution qui change de caractère, avec la possibilité qui se dessine alors de construire un "système juridique » indépendant des sociétés locales, comme cela a été bien décrit par la littérature anthropologique qui traite de l'introduction de l'écrit dans les régions qui furent dominées par la tradition orale (Goody et Watt, 1963; Goody, 1968 ; et surtout Shryock, 1997). Or, cela ne semble pas être le cas en Haute-Égypte. Les documents sont publiés dans une forme schématique et restent étroitement liés aux cas spécifiques auxquels ils se rapportent. L'idée de rassembler ces documents dans un complexe cohérent de textes permettant de les comparer et de les évaluer ne semble pas représenter beaucoup d'intérêt, quand bien même ils sont conservés par un groupe $\mathrm{d}$ 'hommes participant à toutes les assemblées dans la région.

Chaque document est considéré comme le reflet d'un cas simple et unique, la négociation d'un conflit unique, et le savoir qui le sous-tend reste le fait d'individus. Les documents sont des outils mnémotechniques, une réponse au développement accéléré que connaît la société égyptienne ces dernières années et non pas la base du développement d'un édifice juridique complexe et cohérent. 


\section{BIBLIOGRAPHIE}

AMMAR H., 1954, Growing up in an Egyptian Village: Silwa, Province of Aswân, London, Routledge and Kegan Paul.

BOURDIEU P., 1977, Outline of a Theory of Practice, Cambridge, Cambridge U.P.

DRESCH P., 1989, Tribes, Government and History in Yemen, Oxford, Clarendon Press.

GOODY J. (ed.), 1968, Literacy in Traditional Societies, Cambridge, Cambridge U.P.

GOODY J., WATT I., 1962-1963, « The Consequences of Literacy », Comparative Studies in Society and History, $\mathrm{n}^{\circ}$ 5, p. 304-345.

GULLIVER P. H., 1977, « On Mediators », in Hamnett I. (ed.), Social Anthropology and Law, London, ASA Monograph 14.

HOPKINS N. S., 1987, Agrarian Transformation in Egypt, Cairo, AUC Press.

KENNETT A., 1968 (1925), Bedouin Justice: Law and Custom Among the Egyptian Bedouins, London, Frank Cass.

MESSICK B., 1990, "Literacy and the Law: Documents and Document Specialists in Yemen », in Dwyer D. H. (ed.), Law and Islam in the Middle East, New York-London-Wesport, Bergin \& Garvey Publishers.

MOORE S. F. :

- 1977, « Individual Interests and Organizational Structures: Dispute Settlement as « Events of Articulation » ", in Hamnett I. (ed.), Social Anthropology and Law, London, ASA Monograph 14.

- 1995, « Imperfect Communications », in Caplan P. (ed.), Understanding Dispute, Oxford.

SCHACHT J., 1964, An Introduction to Islamic Law, Oxford.

SHRYOCK A., 1997, Nationalism and the Genealogical Imagination. Oral History and Textual Authority in Tribal Jordan, Berkeley, University of California Press.

STEWART F. H., 1987, « Tribal Law in the Arab World: A Review of the Literature », International Journal of Middle East Studies, vol. 19.

\section{NOTES}

1. Cependant il existe sur le Yémen des publications récentes qui traitent du droit coutumier dans un contexte moderne. Voir, par exemple, Dresch (1989) et Messick (1990). Sur le droit coutumier des bédouins en Égypte, voir Kennett (1968) (sur le Sinaï). Sur les habitants de la vallée du Nil, il y a, d'après la bibliographie établie par Stewart (1987), une seule publication: «alTha'r » d'Abû Zayd, publiée en 1965. Il existe néanmoins de courtes descriptions chez Ammar (1954) et Hopkins (1988).

2. Il faudrait ajouter qu'il y a une différence essentielle entre les institutions officielles et coutumières, le prononcé du jugement judiciaire refusant en principe de prendre en considération l'acceptation de son contenu par les parties impliquées ou leur éventuel accord après la décision. Cet élément est essentiel dans les institutions coutumières. Un accord peut toutefois être conclu par l'intermédiaire d'un tribunal officiel. 
3. Par contre, il est bien connu qu'il y a des dissonances entre le 'urf et la législation moderne et nationale, surtout en ce qui concerne le droit de propriété et d'exploitation de la terre.

4. Je fais référence à la distinction entre "médiation » et "adjudication » établie par Gulliver (1977).

5. Ce qui ressort des documents que j'ai en ma possession et qui datent des vingt dernières années. Un seul document (qui traite d'une affaire de succession) remonte aux années cinquante. 6. Les héritages sont distribués selon les prescriptions très détaillées de la sharîa, et les documents qui traitent des affaires de succession sont normalement introduits par un paragraphe par lequel toutes les parties reconnaissent avoir reçu ce qui leur revient de droit. Il peut cependant y avoir désaccord sur la comparabilité des biens - p. ex. : un feddan (0,42 ha) de terre agricole vaut-il un feddan de terrain planté de manguiers, etc.

7. Les chiffres officiels indiquent qu'en 1986, l'analphabétisme s'élevait à $60 \%$ à la campagne et $40 \%$ dans les villes.

INDEX

Mots-clés : droit coutumier, écriture, négociation

\section{AUTEURS}

HANS CHRISTIAN KORSHOLM NIELSEN

Université d'Aarhus 